\title{
Defining and identifying a loading basis for the full-field reconstruction of plate structures under tension loads
}

\author{
Dominique Martini*, Christian Hochard and Jean-Paul Charles \\ LMA (UPR CNRS 7051), 31 chemin Joseph Aiguier, 13402 Marseille Cedex 20, France; Aix-Marseille \\ University, 3 place Victor Hugo, 13331 Marseille Cedex 03, France
}

\begin{abstract}
This method defines a loading basis for plate structures which is identified from strain measurements in order to reconstruct the mechanical fields. This loading basis is given by the decomposition of a global structure into simple substructures associated with the loaded boundaries only. Some elementary bases are defined for each substructure depending on its local edge effect. A global basis is then obtained by the equilibrium of the complete structure. The main advantage of this approach is to classify the basis vectors depending on their influence on the overall response of the structure.
\end{abstract}

Nous proposons la construction d'une base de chargement pour des structures plaques afin de reconstruire les champs mécaniques à partir de mesures de déformation. Cette base de chargement est obtenue par une décomposition de la structure complexe en sous-structures simples associées aux seuls bords chargés. Une méthode de type Trefftz définit des bases de chargement élémentaires en fonction de la localisation des effets de bords induits. La base de chargement globale est ensuite donnée par l'équilibre de la structure complète. L'intérêt est de définir une base de chargement en fonction de son influence sur la réponse globale de la structure.

Keywords: inverse problems; plate structures; structural monitoring; full-field reconstruction

Mots-clés: problèmes inverses; structures plaques; surveillance des structures; econstruction de champs

\section{Introduction}

Improving structural performances requires monitoring the mechanical fields present inside the structures and the boundary conditions. Full-field identification methods have been therefore widely studied for the last 10 years in order to improve the control and the performances of stand-alone structures. Various methods have been implemented in the context of both dynamic and static field reconstructions. The dynamic approaches consisted of identifying modal shapes from strain measurements (Bogert, Haugse, \& Gehrki, 2003; Enzmann, Linz, \& Theis, 1998). The static approaches consisted of identifying finite element fields from strain and displacement measurements (Salzmann, Pilet, Ilic, \& Fua, 2007; Tessler and Spangler, 2005). The main limitation of these methods is the mismatch between the degrees of freedom (DOFs) used to approximate mechanical fields and the less number of measurements available in practical applications. Hundreds of thousands of parameters are required to accurately

*Corresponding author. Email: martini@lma.cnrs-mrs.fr

ISSN 1779-7179 print/ISSN 1958-5829 online

(C) 2012 Taylor \& Francis

http://dx.doi.org/10.1080/17797179.2012.728499

http://www.tandfonline.com 
approximate mechanical fields, whereas only a few sensors are available, which results in an ill-posed inverse problem (Tikhonov and Arsenin, 1977). Since the boundary conditions are used to regularise parameter identification problems on the basis of full-field measurements (Avril et al., 2008), we now propose to first identify the boundary conditions and then to reconstruct the mechanical fields for structural monitoring. In addition, only internal fields are studied, and the corresponding boundary conditions are therefore approximated with just a few parameters based on Saint-Venant's principle. These parameters can therefore be identified with a limited quantity of measurements, which regularises the inverse problem associated with the full-field reconstruction problem. At this point, the main issue is to find a proper loading basis which accurately approximates the real loading conditions with the smallest number of parameters. This basis is obtained by the projection of Trefftz-like analytical solutions of the plate problem onto the boundaries of the structures. In this method, the loading basis is composed of loading functions that are associated with increasing edge effects. The global boundary conditions of the structures are then described with just a few parameters and some functions are simply added when a more precise description of these boundary conditions is required.

This method was first introduced in Martini, Hochard, and Charles (2012) and was applied to the single plates with stress concentration zones. Nevertheless, this application was limited to simple domains only. In this paper, we propose an extension of this method to multi-plate structures under tension loads. This extension is based on a substructuring method. A multi-plate structure consists of an assembly of plates which allows to study complex structures. A global loading basis is then defined from the loading basis defined on each plate substructure using the equilibrium of the whole structure. In the following, we firstly present the inverse problem related to the full-field reconstruction issue. We then illustrate the definition of the loading basis and the resolution of the inverse problem for single plates. Lastly, we show the definition of the loading basis for the multi-plate structures under tension loads and the main results.

\section{Inverse problem formulation}

\subsection{General framework}

The aim here is to determine mechanical fields and boundary conditions based on strain measurements. Let us take a structure $\Omega$ subjected to unknown loading conditions, $F_{\mathrm{b}}$ onto the boundary $\partial \Omega$. At this point, we take the boundary conditions to be loads only and so $F_{\mathrm{b}}$ is necessarily balanced. This assumption, which is required because no displacement measurements are available, makes it possible to avoid the uncertainties about the joints between the structure and the environment. Because of the performance issue, we assume that the structure has an elastic behaviour. Although we illustrate this method with an isotropic elastic behaviour, anistropic behaviour can also be considered. Strain measurements, $\epsilon_{\mathrm{m}}$, are performed on $\partial \Omega_{\mathrm{m}} \subset \partial \Omega$ and the effects of the body force and the inertia are neglected.

Based on the descriptions of inverse problems in Nashed (1987), we decided to define an approximate inverse problem with only a few parameters. Improving the structural performances involves monitoring the internal fields, which mostly depend on the overall effects of the loading conditions, as shown by Saint-Venant's principle. The internal fields can, therefore, be properly reconstructed using some approximate loading conditions such that the overall effects of these approximate and real loading conditions are similar. The structural monitoring problem is, therefore, addressed by identifying the approximate loading conditions, $\bar{F}_{\mathrm{b}}$, and reconstructing the corresponding fields $(\bar{u}, \bar{\epsilon}, \bar{\sigma})$ in $\Omega$. These fields are proper approximations of the real fields $(u, \epsilon, \sigma)$ occurring in a zone of interest $\Omega_{i} \subset \Omega$, which 
corresponds to the internal part of the structure in which the mechanical fields are monitored. This inverse problem is expressed mathematically as follows:

To find $\left(\bar{u}, \bar{\epsilon}, \bar{\sigma}, \bar{F}_{\mathrm{b}}\right)$

$$
\begin{gathered}
\text { Mechanical equations : }\left\{\begin{array}{c}
\operatorname{div}[\bar{\sigma}]=0 \text { in } \Omega \\
\bar{\sigma} \cdot n=\bar{F}_{\mathrm{b}} \text { on } \partial \Omega \\
\bar{\sigma}=\mathbb{C} \bar{\epsilon} \text { in } \Omega
\end{array}\right. \\
\text { Observation equation : } \begin{array}{c}
\bar{F}_{b}=\underset{\arg \min \left[\left\|\bar{\epsilon}\left(\bar{F}_{b}\right)-\epsilon_{m}\right\|^{2}\right]}{\operatorname{an} \partial \Omega_{m} \subset \partial \Omega}
\end{array}
\end{gathered}
$$

\subsection{Finite element approximation}

This problem is solved with the finite element method. Displacement field $u$ is approximated by $\bar{u}(X)=\sum \Phi_{i}(X) \bar{U}_{i}$. The strain field is determined from the strain-displacement relation and the stress field is calculated using the constitutive laws. The loading conditions, $F_{\mathrm{b}}$, are approximated by a $q$-dimension basis, giving $\bar{F}_{\mathrm{b}}=\sum_{i=0}^{q} f_{i} F_{\mathrm{b}}^{i}$ where, $f_{i}$ are the unknown loading parameters and $F_{\mathrm{b}}^{i}$ are some loading functions defined onto the boundary $\partial \Omega$. Discretising the mechanical and observation equations gives the following structural monitoring problem:To find $(\bar{U}, \bar{\epsilon}, \bar{\sigma}, \bar{F})$ such that:

$$
\begin{gathered}
\text { Observation equation } \begin{array}{r}
\bar{F}=\arg \min _{F \in \mathbb{R}^{q}}\left\|\mathbb{B}_{\pi m} U(F)-\epsilon_{m}\right\|^{2} \\
\Leftrightarrow \bar{F}=\arg \min ^{q}\left\|\mathbb{B}_{\pi m} \mathbb{K}^{-1} \mathbb{A} F-\epsilon_{m}\right\|^{2}
\end{array} \\
\text { Mechanical equations : }\left\{\begin{array}{l}
\bar{\epsilon}=\mathbb{B} \bar{U} \\
\bar{\sigma}=\mathbb{C} \bar{\epsilon} \\
\mathbb{K} \bar{U}=\mathbb{A} \bar{F} \Leftrightarrow \bar{U}=\mathbb{K}^{-1} \mathbb{A} \bar{F}
\end{array}\right.
\end{gathered}
$$

where, $\mathbb{K}$ is the stiffness matrix of the structure, $\bar{U}={ }^{T}\left[u_{1}, u_{2}, \cdots, u_{n}\right]$ is the nodal displacement vector and $\bar{F}={ }^{T}\left[f_{1}, f_{2}, \cdots, f_{q-1}, f_{q}\right]$ is the loading parameter vector. Some nodal displacements are fixed in order to avoid the rigid body motion (RBM) and to inverse $\mathbb{K}$. These displacements are chosen not to add reaction forces. The $\mathbb{A}$ matrix is such that:

$$
T \bar{U} A \bar{F}=\int_{\partial \Omega} \bar{u} \cdot \bar{F}_{\mathrm{b}} \mathrm{d} S={ }^{T} \bar{U} \cdot\left[\sum_{i, k} \int_{\partial \Omega} \Phi_{k}(X) F_{\mathrm{b}}^{i}(X) \mathrm{d} S\right] \cdot \bar{F}
$$

$\mathbb{B}_{\pi m}$ is the projection $\Pi_{m}$ of the gradient operator $\mathbb{B}$ onto $\partial \Omega_{\mathrm{m}}$ that gives the relation between $\bar{U}$ and $\epsilon_{\mathrm{m}}$ such that:

$$
\Pi_{\mathrm{m}} \mathbb{B} \bar{U}=\epsilon_{\mathrm{m}} \Leftrightarrow \mathbb{B}_{\pi \mathrm{m}} \bar{U}=\epsilon_{\mathrm{m}}
$$

Lastly, the structural behaviour is assumed to be linear, and the observation equation can therefore be directly solved with the least-squares method. The mechanical fields $\bar{U}, \bar{\epsilon}$, and $\bar{\sigma}$ are then calculated with the mechanical equations. 
This procedure depends on two necessary and sufficient conditions: the number of strain measurements must be greater than the number of loading parameters and $\mathbb{G}=\mathbb{B}_{\pi \mathrm{m}} \mathbb{K}^{-1} \mathbb{A}$ must be a full-rank matrix. The first condition is assumed because the loading conditions are approximated with only a few parameters. The second condition requires that none of the vectors in the loading condition basis is dual with the rigid body motion (RBM). This condition is satisfied because the RBM is only associated with nodal displacements when the loading conditions $\bar{F}_{\mathrm{b}}$ are distributed along $\partial \Omega$. These two conditions being satisfied, $\bar{F}$ is calculated in the least-squares sense with:

$$
\bar{F}=\left({ }^{T} \mathbb{G} \cdot \mathbb{G}\right)^{-1} \cdot{ }^{T} \mathbb{G} \cdot \epsilon_{\mathrm{m}}
$$

The main advantage of this method is that it avoids the instability occurring when increasing the FE DoFs. The identification procedure depends only on the number of loading parameters. The DoFs can therefore be as large as required to ensure the accuracy of the FE model. In addition, as the boundary conditions are all known, the FE mechanical fields can be directly computed using a linear combination of precomputed solutions associated with each of the vectors in the loading basis that is multiplied by the corresponding identified loading parameter. Although FE fields have very large DoFs, these solutions are therefore computed in real time.

\section{Application to a plate under tension loads}

\subsection{Construction of the loading basis}

A basis focusing on the overall response of the structure was constructed to approximate the loading conditions. This construction was based on the Trefftz-like solutions developed in Hochard, Ladevèze, and Proslier (1993) and Hochard (2003) by approximating the displacement field with polynomial functions satisfying the equilibrium equation. The basis of the loading conditions was obtained from the projections of these Trefftz-like solutions onto the boundaries of the structure. The main advantage of this method is that the approximate displacement fields are a complete set of solutions for a given polynomial degree. The projections of these solutions are, therefore, a complete set of loading conditions whose polynomial degrees are associated with increasing edge effects. Consequently, this approach allows to define loading functions that are organised by their influence over the whole structure. Low polynomial degrees that are associated with reduced edge effects are, therefore, used to focus on the overall response of the structure. The corresponding $q-$ degree set of loading conditions is defined as follows:

$$
\begin{gathered}
\mathcal{F}^{q}=\left\{F^{r}=\sigma\left(\bar{u}^{r}\right) \cdot n_{\partial \Omega} ; \bar{u}^{r} \in \mathcal{U}^{q}\right\} \\
\text { with } \mathcal{U}^{q}=\left\{\begin{array}{c}
\bar{u}^{r}=\sum_{j=0}^{r} \alpha_{j r} x^{j} y^{r-j} ; 0<r \leq q \& \operatorname{div}\left[\mathbb{C} \epsilon\left(\bar{u}^{r}\right)\right]=0 \text { in } \Omega \\
\text { and } \Omega \text { is a star - shaped domain }
\end{array}\right\}
\end{gathered}
$$

The vectors of this loading basis are then orthonormalised using the Gramme-Schmidt algorithm in the sense of strain energy. The orthonormalised basis $\mathcal{F}_{\perp}^{q}$ is obtained by:

$$
\begin{aligned}
& \mathcal{F}_{\perp}^{q}=\left\{F_{\perp}^{r} \in \mathcal{F}^{q} ; \forall k<r \int_{\Omega} \operatorname{Tr}\left[\sigma\left(F_{\perp}^{r}\right) \epsilon\left(F_{\perp}^{k}\right)\right] \mathrm{d} \Omega=0 \&\right. \\
& \left.\int_{\Omega} \operatorname{Tr}\left[\sigma\left(F_{\perp}^{r}\right) \epsilon\left(F_{\perp}^{r}\right)\right] \mathrm{d} \Omega=1\right\}
\end{aligned}
$$


In the following, the real loading conditions are approximated by a linear combination of these Trefftz-like loading functions. The loading parameters to be identified are the coefficients that best fit this approximation.

\subsection{Sensitivity of the reconstruction method to the measurement errors}

\subsubsection{Studied case}

Let us take a square plate $\Omega$, showing linear isotropic behaviour, which is subjected to inplane loads, as illustrated in Figure 1. The left boundary is fully clamped and the bottom boundary is linearly loaded. This case cannot be exactly described with the approximate loading conditions because of the clamp.

The sensors are located at the observation distance, $R_{\mathrm{obs}}$, of the centre of the plate and we study the influence of this distance on the measurement error sensitivity. This distance is a convenient way to study the error sensitivity because:

- Since the loading basis is related to the inside fields of the structure, the quality of the approximate inverse problem depends on the distance to the centre of the plate.

- The angular positions of the sensor that minimise the conditioning number of $\mathbb{G}$ are quite the same for every value of $R_{\mathrm{obs}}$.

The measurement errors are of two kinds: modelling errors, $\delta_{\text {mod }}$, corresponding to modelling uncertainties; and random errors, $\delta \epsilon_{\text {rand }}$ corresponding to the data acquisition noise such that: $\epsilon_{\mathrm{m}}=\epsilon_{\mathrm{m}}^{0}+\delta \epsilon_{\mathrm{mod}}+\delta \epsilon_{\mathrm{rand}} . \epsilon_{\mathrm{m}}$ are the measured strains and $\epsilon_{\mathrm{m}}^{0}$ are the real strains. Measurements are simulated with a direct finite element computation associated with the exact boundary conditions. The mechanical fields that correspond to these simulations are called the exact ones. Based on Hochard (2003), a three-degree basis was chosen in order to reduce the quantity of unknowns and sensors. This basis is composed of 15 independent vectors denoted as $\left(F_{\perp}^{r}\right)_{1 \leq r \leq 15}$. The real loading conditions are supposed to be approximated by these 15 functions such that:

$$
\bar{F}_{\mathrm{b}}=\sum_{k=1}^{15} \alpha_{k} F_{\perp}^{k}
$$

$\left(\alpha_{k}\right)$ have then to be identified to obtain the loading vector that is used to compute the nodal displacement in the FEM.
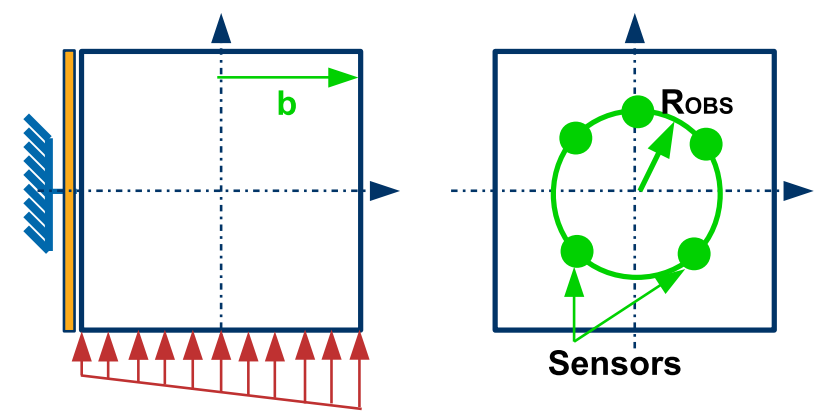

Figure 1. Description of the reference loading case and of the sensor locations. 
Lastly, we consider that each sensor gives three strain values: $\epsilon_{x x}, \epsilon_{y y}$ and $\epsilon_{x y}$. Five sensors are therefore required to identify at least the 15 loading parameters. Only the 5 - sensor configuration is presented here, because additional measurements reduce the sensitivity of the reconstruction method to errors. Lastly, we compare the strain fields of the identified solutions with the exact field using the following expression:

$$
\operatorname{err}(X)=\frac{\operatorname{Tr}\left[\left(\epsilon_{\mathrm{id}}(X)-\epsilon_{\mathrm{ex}}(X)\right) \cdot\left(\sigma_{\mathrm{id}}(X)-\sigma_{\mathrm{ex}}(X)\right)\right]}{\frac{1}{4} \cdot \frac{1}{\Omega} \int_{\Omega} \operatorname{Tr}\left[\epsilon_{\mathrm{ex}}(X) \sigma_{\mathrm{ex}}(X)\right] \mathrm{d} \Omega}
$$

This error is normalised with a reference value of the energy density that corresponds to a threshold such that the energy density of $80 \%$ of the plate is superior to this threshold. In that studied case, this threshold corresponds to a quarter of the mean value of the energy density of the exact case. The main interest of this threshold is to permit the comparison between different loading cases, for example, with or without stress concentration zones.

\subsubsection{Sensitivity to the modelling errors}

The error distributions of the reconstructed fields using the exact measurements are given in Figure 2, using various observation distances. These errors are maximum at the boundaries and minimum at the inside of the structure. This result corresponds to the loading basis definition which is associated with the inside effects of the structure. We also observe that increasing $R_{\mathrm{obs}}$ decreases the errors close to the boundaries and increases the errors at the inside of the structure. Looking then for the largest zone such that the error is less than a chosen threshold, we obtain some optimal observation distances as illustrated in Figure 3. This zone, $A_{\mathrm{ZU}}$, is defined as a percentage of the total area of the structure.

For example, $R_{\mathrm{obs}}=0.65 \mathrm{~b}$ gives a $30 \%$ maximum value of $A_{\mathrm{ZU}}$ such that the error is less than $10 \%$. Supplementary loading cases show that the clamp condition is a very severe case and that the optimal observation distances remain the same depending on the error threshold. Only the $A_{Z U}$ value is changed with these other loading cases. This result shows that the loading basis is relevant to approximate the global effects of the real loads applied to the structure.

\subsubsection{Sensitivity to the random errors}

The random errors are associated with the measurement noise resulting in the measurement acquisition. The sensitivity of the reconstruction method to these errors is estimated using the standard deviation ratio, $\tau_{\mathrm{STD}}$ which is defined by:

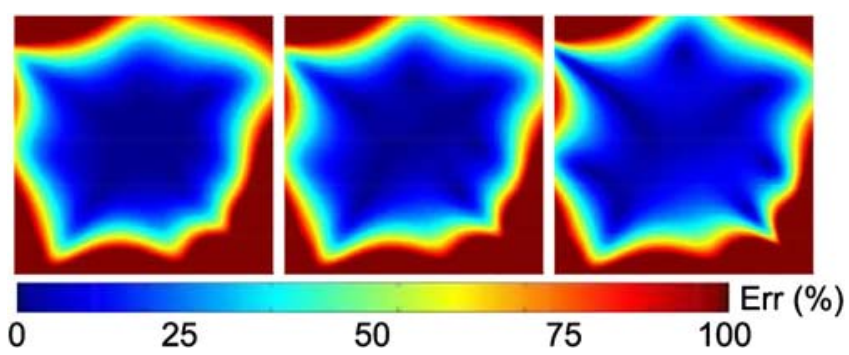

Figure 2. Distributions of the error of the reconstructed solutions with different observation distances using unnoisy measurements. From left to right, $R_{\text {obs }}$ is equal to $0.3 b, 0.5 b$ and $0.7 b$. 


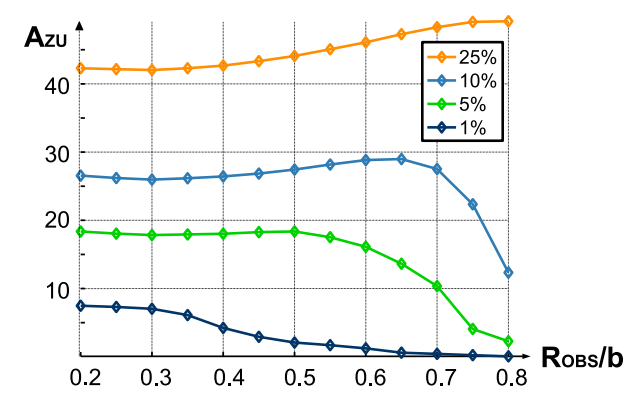

Figure 3. Evolution of the area of the interest zone, $A_{\mathrm{ZU}}$, as a function of $R_{\mathrm{obs}}$ with different values of the error threshold.

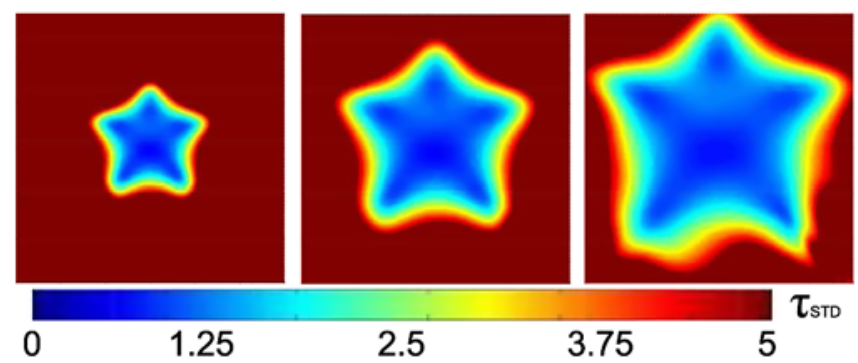

Figure 4. Distributions of the standard deviation ratio $\tau_{\mathrm{STD}}$ of the reconstructed solutions with different observation distances in the case of noisy measurements. From left to right, $R_{\mathrm{obs}}$ is equal to $0.3 b, 0.5 b$ and $0.7 b$.

$$
\tau_{\mathrm{STD}}=\frac{\langle\langle\operatorname{err}(X)\rangle\rangle_{N}}{\left\langle\left\langle\epsilon_{\mathrm{m}}\right\rangle\right\rangle_{N}}
$$

$\langle\langle\cdot\rangle\rangle_{N}$ is the standard deviation computed with $N$ samples. This ratio shows the variation of the solution due to the measurement noise. Figure 4 illustrates the distributions of this ratio with different values of the observation distance. This ratio is less than 1 inside the observation zone, which means that the variation of the solution is equivalent to the variation of the measurement due to the noise. This ratio then increases at the outside of the observation zone within the limit of $\tau_{\mathrm{STD}} \leq$ Cond $[\mathbb{G}]$ at the boundaries of the structure. This result shows that the mechanical fields are reliably reconstructed in the observation zone.

\subsubsection{Optimal sensor location}

These previous results help to address the optimal sensor location issue. We define the optimal sensor locations, such that the sensitivity to the errors is minimised in the largest interest zone. Consequently, the optimal sensor locations are a compromise between the sensitivity to the modelling errors and the sensitivity to the measurement errors. The first one tends to decrease $R_{\mathrm{obs}}$ when the second one tends to increase it, as shown in Figures 3 and 4 . Consequently, the optimal sensor locations are defined depending on the most restrictive errors. For example, if the modelling error is the most influent error, then $R_{\mathrm{obs}}$ is decreased in order to maximise the size of the interest zone and the sensitivity to the measurement errors increases. 
At the opposite, if the measurement error is the most influent error, then $R_{\mathrm{obs}}$ is increased and the precision of the solution decreases. Lastly, when the modelling and the measurement errors are similar, $R_{\mathrm{obs}}$ is approximatively equal to half of the distance between the centre of the plate and the boundaries.

\section{Extension to plate structures under tension loads}

\subsection{General approach}

At this point, the main limitation of the proposed method is the restriction of the loading basis to simple domain only. We now propose an extension of the loading basis, which consists of defining elementary substructures of the complete structure depending on the boundaries having unknown loads only. This extension allows to generalise our method to any kind of plate structures under complex loads. Trefftz-like loading bases are then defined for each of these elementary substructures and the global loading basis is obtained from the equilibrium of the complete structure. In the following, we limit our study to tension loads but a similar procedure can be used for complex loads. Figure 5 illustrates the definition of these substructures in the case of three boundaries with unknown loads, $\partial \Omega_{1} \cap \partial \Omega, \partial \Omega_{2} \cap \partial \Omega$ and $\partial \Omega_{3} \cap \partial \Omega$ of the structure $\Omega$. The remaining part of $\partial \Omega$ is free from loading. Noting $F_{i}$, the unknown loading condition of the boundary $\partial \Omega_{i}$, and $\left(F_{b i}^{k}\right)_{1 \leq k \leq p_{i}}$, the loading basis of the substructure $\Omega_{i}$, the global equilibrium of the complete structure is expressed by:

$$
\left\{\begin{array}{l}
\sum_{i=1}^{r} \sum_{k=1}^{p_{i}} \int_{\partial \Omega \cap \partial \Omega_{i}} \alpha_{k}^{i} F_{b i}^{k}=0 \\
\sum_{i=1}^{r} \sum_{k=1}^{p_{i}} \int_{\partial \Omega \cap \partial \Omega_{i}} \alpha_{k}^{i} O P \wedge F_{b i}^{k}=0
\end{array} \Leftrightarrow \mathbb{U} \cdot F=0\right.
$$

$\wedge$ is the vectorial product that is used to obtain the moment equation. The loading basis $\mathcal{F}_{\perp}^{T}$ of the complete structure is then defined by the kernel of $\mathbb{U}$ and orthonormalised with respect to the strain energy:

$$
\mathcal{F}_{\perp}^{T}=\left\{F_{\perp}^{r} \in \operatorname{Ker} \llbracket ; \forall k<r \int_{\Omega} \operatorname{Tr}\left[\sigma\left(F_{\perp}^{r}\right) \epsilon\left(F_{\perp}^{k}\right)\right] \mathrm{d} \Omega=0 \&\right.
$$

\subsection{Application to an $L$ - structure}

Let us consider the L-structure $\Omega$. The top edge is fully clamped and two edges on the left are linearly loaded, as illustrated in Figure 6. These displacement and loading conditions are supposed to be unknown and associated with the unknown loads $F_{1}$ and $F_{2}$, and the two

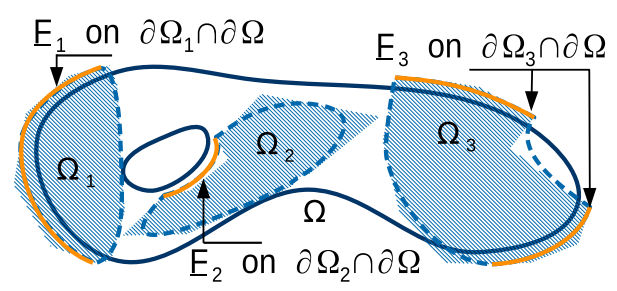

Figure 5. Illustration of the complete plate structure $\Omega$ and of the substructures $\Omega_{1}, \Omega_{2}$ and $\Omega_{3}$, respectively associated with the boundaries $\partial \Omega_{1}, \partial \Omega_{2}$ and $\partial \Omega_{3}$ having unknown loads. 
substructures $\Omega_{1}$ and $\Omega_{2}$ are defined by the corresponding boundaries, as shown in Figure 6 . The loading basis of the complete structure is obtained by the two Trefftz-like bases of each substructure.

The sensitivity of the reconstructed fields to error measurements is then studied depending on the sensor locations. The dimension of the global loading basis is 14 and only five sensors are needed to identify the loading parameters. We observe that a necessary condition in order to minimise the conditioning number of $\mathbb{G}$ and the sensitivity to the modelling errors is that the sensors are located in the elementary structures with the unknown loading boundaries. Moreover, these substructures can be individually considered for the sensor location. The optimal sensor location is obtained with the same approach as for the plate inverse problem.

Figure 7 shows the distribution of the error of the reconstructed solutions without noise. We observe that the error is minimal in the structure and also at the unloaded boundaries.
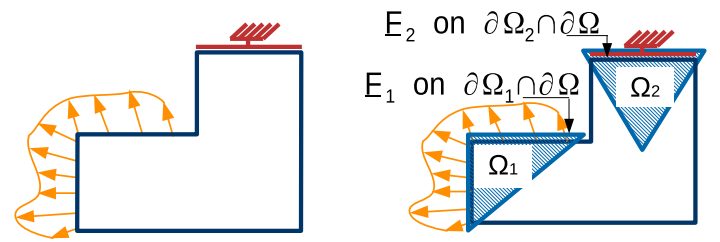

Figure 6. Illustration of the $L-$ structure $\Omega$ with its loading conditions and illustration of the elementary substructures associated with the boundaries $\partial \Omega_{1}$ and $\partial \Omega_{2}$ having unknown loads $F_{1}$ and $F_{2}$.

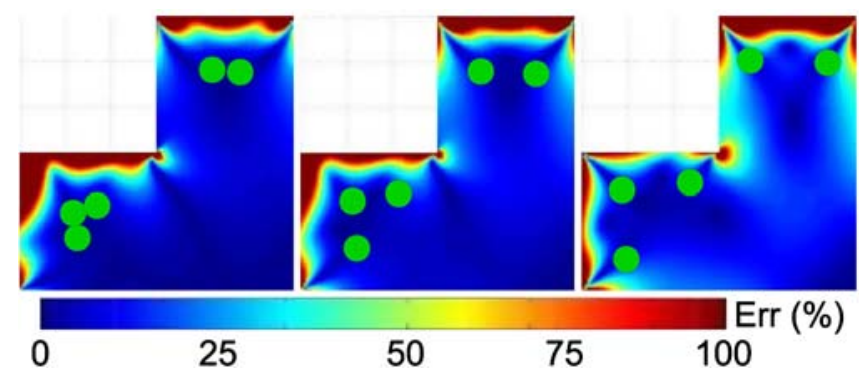

Figure 7. Distributions of the error of the reconstructed solutions with different observation distances and using unnoisy measurements. The green circles show the sensor locations in each substructure $\Omega_{1}$ and $\Omega_{2}$.

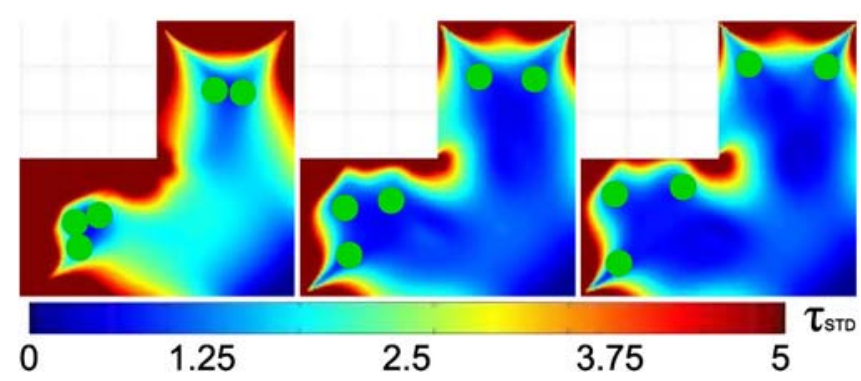

Figure 8. Distributions of the standard deviation ratio $\tau_{\mathrm{STD}}$ of the reconstructed solutions with different observation distances and using noisy measurements. The green circles show the sensor locations in each substructure $\Omega_{1}$ and $\Omega_{2}$. 
These results show the interest of the proposed loading basis that localises the uncertainties of the model at the boundaries with the unknown loads only. At the opposite, the inside mechanical fields are accurately reconstructed. These results also give an optimal observation distance depending on the error threshold that is similar to the one-plate case.

Figure 8 shows the distribution of the standard deviation ratio $\tau_{\mathrm{STD}}$ with different observation distances. The proposed loading basis is therefore a proper approximating basis for the loading conditions: the errors of the reconstructed fields are similar to the measurement errors at the inside of the structure and the maximum errors are localised at the loaded boundaries.

\section{Conclusion and prospects}

In this paper, we showed that the full-field reconstruction can be reduced to a load identification problem. The unknown loads are described with just a few parameters in comparison with the unknown mechanical fields because overall information about the structure is required for structural monitoring purposes such as command and performances. We then proposed a method for defining a loading basis for single-plate and multi-plate structures in order to reconstruct the mechanical fields from the loading parameters identification. This loading basis is associated with the overall response of the structure based on analytical Trefftz-like solution. Overall information is accurately recovered at the inside of the structure because the effects of the modelling and the measurement errors are localised at the boundaries with the unknown loads. Lastly, further work is required in order to precisely estimate the absolute errors of the solutions. This definition will help to optimise the sensor locations, which is also a major issue when the available measurement information is limited.

\section{References}

Avril S., Bonnet M., Bretelle A., Grédiac M., Hild F., Ienny P., ... Pierron F. (2008). Overview of identification methods of mechanical parameters based on full-field measurements. Experimental Mechanics, 48(4) 381-402.

Bogert, P.B., Haugse, E., \& Gehrki, R.E. (2003). Structural shape identification from experimental strains using a modal transformation technique. In 44th AIAA/ASME/ASCE/AHS/ASC structures, structural dynamics and material conference and exhibit, 7-10 April 2003, Norfolk, AIAA Inc.

Enzmann, M., Linz, C., \& Theis, T. (1998). Robust shape control of flexible structures using strain-measurement-gauges and piezoelectric stack actuator. In 4th ESSM and 2nd MIMR conference, 6-8 July 1998, Hurrogate, 123-130.

Hochard, Ch. (2003). A Trefftz approach to computational mechanics. International Journal for Numerical Methods in Engineering, 56(15), 2367-2386.

Hochard, Ch., Ladevèze, P., \& Proslier, L. (1993). A simplified analysis of elastic structures. European Journal of Mechanics A Solids, 12(4), 509-535.

Martini, D., Hochard, Ch., \& Charles, J.-P. (2012). Load identification for full-field reconstruction: applications to plates under tension loads. International Journal of Numerical Method in Engineering. doi:10.1002/nme.4304

Nashed, Z. (1987). A new approach to classification and regularization of ill-posed operator equations. In H.W. Eng \& C.W. Groetsch (Eds.), Inverse and ill-posed problems. Boston: Academic Press.

Salzmann, M., Pilet, J., Ilic, S., Fua, P. (2007). Surface deformation models for nonrigid 3D shape recovery. IEEE Transactions on Pattern Analysis and Machine Intelligence, 29(8), 1481-1487.

Tessler, A. \& Spangler, J.L. (2005). A least-squares variational method for full-field reconstruction of elastic deformations in shear-deformable plates and shells. Computer Methods in Applied Mechanics and Engineering, 194(2-5), 327-339.

Tikhonov, A.N., \& Arsenin, V.Y. (1977). Solution of ill-posed problems. New York: Winston. 